\title{
Zukunft der Bedarfsplanung und Gestaltung der
} Versorgung

Im Jahre 2011 wird die Gesundheitspolitik in Deutschland mit dem sogenannten „Versorgergesetz “ die Reform der ärztlichen Bedarfsplanung ansteuern, die Abgrenzung zwischen ambulanter und stationärer Versorgung neu zu ordnen beginnen und ggf. eine erneute Honorarreform im ärztlichen Bereich in Angriff nehmen. Dafür sind aus Sicht der Krankenkassen auch Grundsatzentscheidungen unabdingbar, mit denen sich der Wettbewerb nicht länger auf die Seite der Krankenkassen beschränkt, sondern gerade auf der Versorgungsseite zu mehr Stabilität, besseren Leistungen und mehr Qualität beiträgt. Erneut „mehr Geld“ ins System zu bringen, kann nicht das Ziel sein. Vielmehr richten die befristeten Ausgabenbegrenzungen des GKV-Fin G den Blick auf inhaltliche Reformen der ambulanten und stationären Versorgung. Nach Jahren zunehmender Zentralisierung und Vereinheitlichung der vertraglichen Gestaltung des Gesundheitswesens bedarf es zudem dringend mehr dezentraler Elemente, um innerhalb des Gesundheitsfonds die erforderliche Handlungsfähigkeit der Krankenkassen wieder herzustellen. Von daher wird mit diesem Papier um den Kern einer neuen Bedarfsplanung herum ein umfassenderes Reformprogramm diskutiert.

\section{K.H. Schönbach, Birgit Schliemann, Jürgen Malzahn, Jürgen Klauber, Dr. Christian Peters}

\section{Ausgangslage}

\section{Finanzielle Stabilisierung der GKV}

Mit dem GKV-Finanzierungsgesetz (GKV-FinG) wendet der Gesetzgeber ein ansonsten für das Jahr 2011 drohendes Defizit der GKV in Höhe von 11 Mrd. Euro ab. Das erwartete Defizit wurde nicht primär durch die aktuelle Finanzkrise begründet, sondern durch eine übermäßige Zunahme der Ausgaben. Seit dem Jahre 2009 eilt die Ausgabenentwicklung der Entwicklung der beitragspflichtigen Einnahmen der GKV bzw. des Bruttoinlandsprodukts verstärkt voraus.

Höhere Beitragssätze und Steuerzuschüsse sind die Folge. Ermöglicht wurde die Ausgabenentwicklung durch die Gesetzgebung der Großen Koalition in der 16. Legislaturperiode. Mit ihr haben sich „medizinische Orientierungsdaten“ für die Weiterentwicklung der Ausgaben gegenüber zuvor weithin „ökonomischen Orientierungsdaten“ nahezu vollständig durchgesetzt. Zuvor wirksame „ökonomische Orientierungsdaten“ wurden zurückgedrängt und beschränken sich weithin auf die zusätzliche Fortschreibung berücksichtigungsfähiger Kostenanteile. Nach geltendem

Die Autorinnen und Autoren sind im Geschäftsbereich Versorgung des AOK-Bundesverbandes bzw. dessen Wissenschaftlichen Institut in Berlin tätig.
Recht sind die entscheidenden Treiber der Ausgabenentwicklung inzwischen

- die „Innovationsquote“ bei den Arzneimitteln,

- die „Morbiditätsquote“ bei den Arztausgaben und

- der Anstieg der „Case-Mix-Punkte“ bei den Krankenhausausgaben.

Diese aus dem Medizinbetrieb selbst abgeleiteten Treiber unterliegen zahlreichen Einflüssen und sind in hohem Maße variabel. So brachten Mengen- und Struktureffekte des Medizinbetriebes zuletzt durchschnittliche Ausgabenzuwächse von jährlich $6 \%$ und mehr hervor, während

- die Bevölkerungszahl bzw. die Zahl der Versicherten stagniert,

- der in den alters- und geschlechtsspezifischen Ausgabenprofilen gemessene demographisch bedingte Ausgabenanstieg 0,2 Prozentpunkte pro Jahr beträgt,

- die Entwicklung des Lohn- und Preisniveaus überaus stabil ist,

- medizinische Entwicklungen, die solche Zuwachsraten erklären, kaum beobachtet werden können.

Aus diesem Grunde hat die Bundesregierung für die mit dem GKV-FinG auf zwei Jahre befristeten Ausgabenbegrenzungen breite politische Unterstützung gefunden. Auch 
Abb. 1: Ausgabenentwicklung der GKV löst sich von der Einnahmenentwicklung

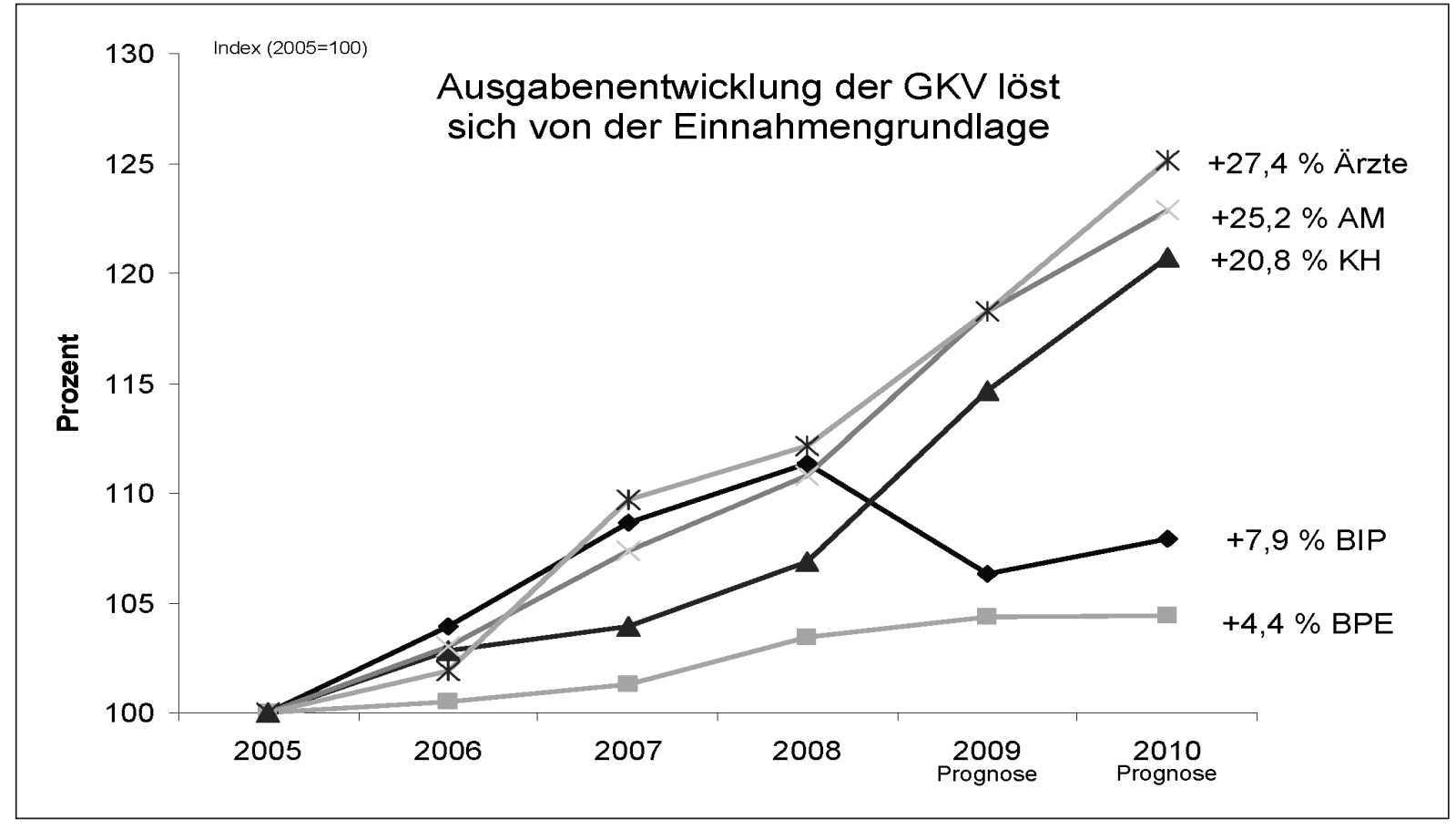

Quelle: Amtliche Statistik und Prognose des Schätzerkreises beim BMC

„Zusatzbeiträge“ würden solchen Ausgabensteigerungen künftig nicht standgehalten können. Daher hat die Bundesregierung angekündigt, während der kommenden zwei Jahre strukturell wirksame Reformen einzuleiten, die zu mehr Solidität der Ausgabenentwicklung beitragen. Als ein erstes wichtiges Vorhaben hierzu hat sie das AMNOG auf den Weg gebracht, das insbesondere die preisliche Markteinführung neuer Arzneimittel nach dem Zusatznutzen differenzieren und Preisverhandlungen für patentgeschützte Arzneimittel ermöglichen soll.

\section{Wettbewerbliche Strukturreformen in der ambulanten Versorgung}

Über das AMNOG hinaus sollen mit dem hier vorgelegten Papier insbesondere für sektorunabhängige ambulante Leistungen der spezialärztlichen Versorgung Eckpunkte aufgezeigt werden, wie durch strukturelle Reformen mehr Stabilität erreicht werden kann. Auch allgemein weist die ambulante ärztliche Versorgung zunehmende Instabilität und Steuerungsdefizite auf, die für diese Drehscheibe der gesamten GKV inakzeptabel sind. Obwohl für die Vergütung der niedergelassenen Ärzte allein in den Jahren 2008, 2009 und 2010 rund 5 Mrd. Euro zusätzlich aufgebracht wurden, kehrt an der Vergütungsfront keine Ruhe ein. Die ärztliche Selbstverwaltung bezeichnet das mittlerweile vielfältig überarbeitete Honorarsystem als nicht weiter reformierbar. Daher hat die Bundesregierung bereits für das kommende Jahr ein „einfaches und gerechtes System“ angekündigt. Dabei sind die „ärztliche Primärversorgung“ und die „allgemeine fachärztliche Versorgung“ von der „spezialärztlichen Versorgung“ zu unterscheiden. Während die Primärversorgung und die allgemeine fachärztliche Versorgung in erster Linie nach Regelleistungsvolumen (RLV) aus der „Morbiditätsbedingten Gesamtvergütung“ (MGV) finanziert werden, ist die spezialärztliche Versorgung - mit teilweise parallelen Sicherstellungsbeiträgen der Krankenhäuser - weithin durch Einzelleistungen außerhalb der MGV (EGV) vergütet.

\section{Vergütung innerhalb der Regelleistungsvolumina}

Auf einer neuen gesetzlichen Basis soll die Rückkehr zu einem „einfachen und gerechten“ Honorarsystem erfolgen (§ 87 Abs. 9 SGB V). Von daher scheidet eine weitere Ausdifferenzierung der gesetzlichen Regelungen zur „Morbiditätsbedingten Gesamtvergütung“ (MGV) aus. Diese Regelungen haben einen Grad der Komplexität erreicht, der das Ergebnis von Änderungen weithin einer Prognose entzieht. Zudem würden die Krankenkassen als Ergebnis eines so genannten Aufteilungsmodells die jeweiligen Zuweisungen aus dem Risikostrukturausgleich - ohne jede Chance ihrer wettbewerblichen Beeinflussung - an die Kassenärztlichen Vereinigungen verteilen. Der ordnungspolitische Zweck des Risikostrukturausgleichs, unverzerrten Wettbewerb erst zu ermöglichen, würde beeinträchtigt. Eine kostentreibende Finanzausgleichs- und Verteilungsmentalität würde Platz greifen. 
Aus den genannten Gründen sollte eine Stärkung föderaler Elemente bevorzugt werden. Dazu sind zentrale Elemente der Honorarverteilung abzubauen und die Verhandlung des Volumens der Gesamtvergütungen aufgrund unterschiedlicher sektorübergreifender Strukturen wieder subsidiär in die Hände der Gesamtvertragspartner zu legen. Dazu bedarf es zweifellos auch Rahmenvorgaben auf Bundesebene. Nur aber wenn wieder im Land selbst „verhandelte“ Verträge die regionalen Strukturen angemessen berücksichtigen können, wird Stabilität ermöglicht. Die föderale Struktur eines auf Verständigung beruhenden Vertragssystems der GKV ist mit den zurzeit geübten Entscheidungsverfahren des Erweiterten Bewertungsausschusses kaum vereinbar. Auch eine valide Messung der ambulanten Morbiditätsentwicklung muss als ein Element in die Verhandlungen der Gesamtvertragspartner eingehen. Bisher wurden Werte als Ausdruck der Morbiditätsentwicklung ergebnisorientiert vorgegeben. Dies wird der Funktion des Bewertungsausschusses nicht gerecht. Er sollte künftig wieder auf die Aufgabe konzentriert werden, Vergütungsrelationen der Leistungen des EBM zuverlässig zu bestimmen und ggf. neue Leistungen in den EBM „einzupreisen“. Im Gegensatz dazu müssen das Ausbalancieren des Tarifniveaus und der Verteilung von den Gesamtvertragspartnern selbst entschieden werden.

\section{Vergütung nach Einzelleistungen}

Gerade im Bereich der „spezialärztlichen Versorgung“ - im Kern Gegenstand des hier vorgelegten Konzepts - ist mehr Stabilität durch eine strikte Qualitätsorientierung anzustreben. Die über die haus- und fachärztliche Grundversorgung hinausgehende spezialärztliche Versorgung der Vertragsärzte konkurriert sektorübergreifend mit der entsprechenden ambulanten Behandlung am Krankenhaus. Die hier erbrachten Leistungen sind weithin in Form der Einzelleistungsvergütung (EGV) außerhalb der morbiditätsbedingten Gesamtvergütung unbegrenzt finanziert. Die EGV-Steigerungsrate hat sich vorerst von durchschnittlich 10\% im Jahre 2009 auf rund 6\% im Jahre 2010 abgeflacht, wird aber weiter angebotsinduziert geprägt. Daher will die Bundesregierung den Anstieg der Einzelleistungsvergütung an dieser Stelle mit den ökonomischen Instrumenten des GKV-FinG noch deutlicher bremsen, als den der MGV. Der weitere Anstieg soll jährlich höchstens 0,9\% betragen. Dabei bleiben die konkreten Begrenzungsmaßnahmen den Gesamtvertragspartnern vorbehalten.

Von daher scheint es unverzichtbar, dass innerhalb der Zwei-Jahres-Frist des GKV-FinG gerade für die spezialärztliche Versorgung strukturelle Reformen vorbereitet werden. Und wenn es richtig ist, dass die Vergütung spezialärztlicher Leistungen nicht dauerhaft abgestaffelt werden kann, weil sie nicht a priori variabel sind und im konkreten Einzelfall ggf. erbracht werden müssen, bedarf es neuer Lösungen, um eine qualitätsgesicherte und wirtschaftliche Versorgung mit spezialärztlichen Leistungen sicherzustellen.
Für Steuerungsoptionen bieten sich sektorübergreifende Versorgungsverträge unter Leistungsbezug an. Je spezifischer eine Leistung medizinisch mit einem konkreten Patientenproblem verbunden und damit von der Indikationsstellung her ableitbar ist, desto eher kann ihr auch ein populationsbezogener Bedarf zugeordnet werden. Dieser positive Zusammenhang gilt auch für eine angemessene Vergütung und die Qualitätskonzepte.

\section{Perspektive: Indikationsbezogene Vergütung}

Die ad hoc geführte Diskussion um ein „transparentes und verständliches" Vergütungssystem in der ärztlichen Versorgung ist im bestehenden System mit EBM nicht durch das Umlegen von ein oder zwei Schaltern zu beantworten. Da der EBM bei fachärztlichen Leistungen eher (Teil-) Prozeduren beschreibt und (noch) nicht indikationsbezogen an komplexen Patientenstichproben anknüpft, bietet er ohne Begrenzungen die Grundlage für eine beliebige Mengenentwicklung. Dies ist der Grund, warum Gesetzgeber aller Couleur verschiedene Plafonds, Budgets, Regelleistungen usw. vorgegeben haben. Gerade komplexe fachärztliche und spezialisierte Leistungen verlangen aber nach „diagnosebezogenen Fallpauschalen“ wie sie ähnlich im stationären Sektor eingeführt wurden. Für die Morbiditätsorientierung der ambulanten ärztlichen Versorgung wurde aber methodisch nicht in die Frage der Verteilung der Mittel investiert, sondern nur in die der Aufbringung. Der Morbiditätsbezug soll Finanzierungsbedarf begründen, die Gesamtvergütung wird aber nicht der Morbidität der Patienten nach verteilt.

Mit der Ablösung des EBM durch ein Vergütungssystem mit Pauschalen, indikationsbezogenen Komplexgebühren und verbleibenden Einzelleistungsvergütungen ließe sich im Kern das Prinzip primär indikationsbezogener, kostenhomogener Fallpauschalen realisieren. Da die Kodierung von Diagnosen (und ggf. auch Prozeduren) eine zwingende Voraussetzung für die Ermittlung ambulanter Fallpauschalen ist, resultiert ein hoher Grad an Transparenz bei „Morbidität“ und Leistungsgeschehen. Ein im Kern indikationsbezogen arbeitendes Vergütungssystem ließe sich anders als ein System mit „Pauschalen je Fall“ sowohl im Rahmen kollektiver wie auch direkter Versorgungsverträge anwenden. Die Kostengewichte der indikationsbezogenen Fallpauschalen würden vom Institut des Bewertungsausschusses (InBA) bundesweit einheitlich definiert, die konkreten Vergütungshöhen durch die Bemessung des Basisfallwertes ausgedrückt. Sowohl in kollektivem als auch direktem Vertragsrahmen erhalten die Vertragspartner auf diese Weise die Möglichkeit, Vergütungsvolumina durch die Verhandlung der Basisfallwerte zu vereinbaren.

\section{Perspektive: Wettbewerb mit Qualitätsvorrang}

Insbesondere bei komplexen fachärztlichen und spezialisierten Leistungen sind auch Qualität und rechtzeitiger Zugang zu einem qualifizierten Arzt für die Versicherten individuell von entscheidender Bedeutung. Ein rechtzei- 
tiger Termin beim Spezialisten kann Krankenhausaufenthalte vermeiden. Die zutreffende Diagnose einer seltenen Erkrankung kann den Leidensweg des Patienten entscheidend verkürzen. Ein erfahrender ambulanter Operateur verringert die Wahrscheinlichkeit von Folgeinterventionen. Da das „Ob und Wie“ einer medizinischen Bedarfsdeckung für eine individuelle Nachfrage im Krankheitsfall überaus variabel ist, sind auch der Mengenexpansion im Medizinbetrieb kaum Grenzen gesetzt. Wo der Arzt ehedem als Anwalt des Patienten den Krankheitsverlauf wenn möglich erst einmal beobachtet hat, wenn kein akuter Interventionsbedarf vorlag, wird die Palette des medizinisch Möglichen heute oft unmittelbar in Gang gesetzt und ökonomisch ausgereizt. Der viel beschriebenen Ökonomisierung der Gesellschaft kann sich auch der Medizinbetrieb oft nicht entziehen. Anreize, mit immer mehr Leistungen um Vergütungen zu konkurrieren, werden von Standesvertretern öffentlich eingeräumt. Nirgendwo in der OECD ist die Großgerätedichte höher als in Deutschland, erfolgen mehr Katarakt-Operationen, werden so zahlreiche Herzkatheter-Untersuchungen durchgeführt, Arthroskopien, ambulante MRTs usw. durchgeführt: Die medizinische Angemessenheit der Leistungshäufigkeit wird dabei auch von vielen Ärzten mit Skepsis beurteilt. Nach verschiedenen Erhebungen liegt die Häufigkeit von Operationen im Durchschnitt ein Drittel höher als bei Ärzten und ihren Angehörigen.

Während - bei im konkreten Einzelfall ggf. unverzichtbaren Leistungen - „Rationierung“ ausscheidet, spricht gegen ökonomische Anreize zur Ausdehnung von Leistungen neben einer nachdrücklichen Evidenzbasierung der Medizin in erster Linie das Interesse der Patienten, Schäden durch Über- und Fehlbehandlung zu vermeiden. Mit anderen Worten: Indikationsstellung und Qualitätssicherung nehmen für die Versicherten an Stellenwert zu. Dieses Interesse der Versicherten erhöht zunächst die Bereitschaft, sich zu informieren und den Arzt zumindest beim ersten Besuch unter Zuhilfenahme ergänzender abgesicherter Informationen aufzusuchen. Sind konkrete Versorgungsaufträge vereinbart, kann die Krankenkasse ihren Versicherten Informationen, Beratung und Terminmanagement zukommen lassen bzw. Hilfestellungen im Rahmen von Angebotsnavigatoren anbieten.

Die Herausforderung besteht mithin im Qualitätswettbewerb. Keiner der Akteure wird sich anmaßen, diese Herausforderung mit wenigen Instrumenten zu meistern. Längst räumen auch die Kassenärztlichen Vereinigungen ein, dass „Sicherstellung“ nur sektorübergreifend zu leisten ist. Es bedarf mithin ordnungspolitisch fundierter Reformansätze, die sich mit der Diskussion um eine neue, sektorübergreifende Bedarfs- bzw. Versorgungsplanung verbinden. Diese Einordnung wird im nächsten Abschnitt geleistet werden.

\section{Versorgungsplanung}

\section{Rahmenbedingungen der gegenwärtigen Bedarfsplanung}

Die Bedarfsplanung ist zurzeit zu starr und inflexibel, um den sich kontinuierlich verändernden Versorgungsbedarfen gerecht zu werden. Diese ergeben sich insbesondere durch demografische Veränderungen, Wanderungsbewegungen der Bevölkerung weg von infrastrukturschwachen Gebieten hin zu Ballungsräumen sowie eine Veränderung des Morbiditätsspektrums. Auch das Durchschnittsalter der Ärzte insbesondere in der hausärztlichen Versorgung ist zu berücksichtigen. Zudem erfordert die fortgeschrittene Spezialisierung der Medizin bei komplexen fachärztlichen Leistungen allein aus Gründen der Qualitätssicherung Teamstrukturen, wie sie an spezialisierten Zentren, MVZs und Krankenhäusern vorgefunden werden.

Der aktuellen Bedarfsplanung gelingt es nicht, Arztpraxen dort anzusiedeln, wo ein lokaler Versorgungsbedarf besteht. Zudem hat der durch das Vertragsarztrechtsänderungsgesetzt (VÄndG) ausgelöste „Liberalisierungsschub“ dazu geführt, dass zunehmend Fakten geschaffen werden können, die sich einer Planung entziehen. Die Folge sind gelegentliche Versorgungslücken einerseits und zahlreiche überversorgte Gebiete andererseits. In Versorgungslücken fehlen zurzeit insgesamt 800 Ärzte, während in überversorgten Gebieten 25.000 Ärzte über den Bedarf hinaus tätig sind. Vor diesem Hintergrund bedarf es vor allem eines flexiblen Raumkonzepts, mit dem die Bedarfsplanung auf lokale Disparitäten und Bedarfe an bestimmten Arztgruppen reagieren kann.

Darüber hinaus wird eine sektorübergreifende Planung, die nicht substituierend, sondern im Verständnis einer Rahmenplanung fachärztliche Versorgung sicherstellt, von den Beteiligten überwiegend begrüßt. Dazu bedarf es Planungsgremien auf Bundes- und Landesebene, die alle Beteiligten einbeziehen:

- Im Unterausschuss Bedarfsplanung des Gemeinsamen Bundesausschusses (G-BA) sollten die Länder ein Mitberatungsrecht haben.

- Für die Landesausschüsse sollte es bei der bisherigen Zusammensetzung bleiben. Die Vertretung der Arztseite wird aber in eine gemeinsame Vertretung aus Kassenärztlicher Vereinigung und Landeskrankenhausgesellschaft zu überführen sein.

Eine solche Anpassung der Zuständigkeiten und Vertretungsrechte in den Gremien der Selbstverwaltung erlaubt, die Hürden, die mit dem geteilten Sicherstellungsauftrag verbunden sind, zu überwinden und eine sektorenunabhängige Versorgungssteuerung zu ermöglichen. Die Aufsichtsrechte und das politische Zusammenwirken aller für die Daseinsvorsorge Verantwortlichen bleiben unberührt.

Eine einheitliche sektorenunabhängige Rahmenplanung wird allerdings grundlegend erschwert durch unterschiedliche Zulassungssysteme für Vertragsärzte und Ärzte im 
stationären Bereich, die an der ambulanten Versorgung teilnehmen.

\section{Flexibilisierung der Bedarfsplanung - Neue Versorgungsplanung}

Aus den Defiziten der gegenwärtigen Bedarfsplanung sind die Anforderungen an eine neu zu gestaltende Versorgungsplanung abzuleiten:

\begin{tabular}{|c|c|}
\hline Kurzfristige Neuausrichtung & $\begin{array}{l}\text { Strukturelle Wettbewerbsori- } \\
\text { entierung }\end{array}$ \\
\hline 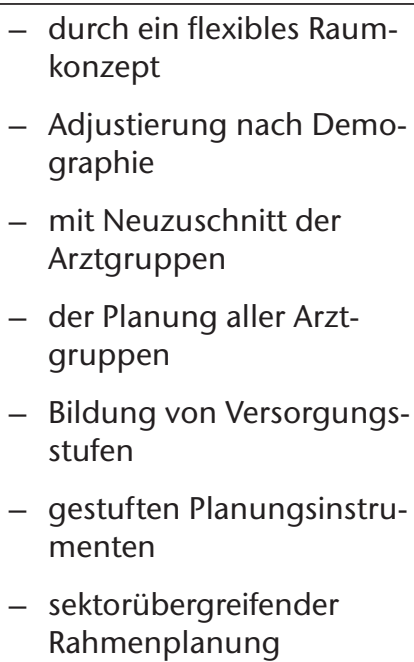 & $\begin{array}{l}\text { - Vereinheitlichte Zulas- } \\
\text { sung für die Teilnahme an } \\
\text { der ambulanten Versor- } \\
\text { gung } \\
\text { - Direkte Verträge im } \\
\text { Bereich der spezialisierten } \\
\text { Leistungen zur Sicher- } \\
\text { stellung des Bedarfs der } \\
\text { Versicherten einer Kran- } \\
\text { kenkasse }\end{array}$ \\
\hline
\end{tabular}

\section{Intelligente Planungsräume - Flexibles Raumkonzept}

Ein flexibles Raumkonzept setzt voraus, dass den Landesausschüssen vor Ort Variablen zur Verfügung stehen, um Räume anforderungsgerecht zu bestimmen und flexibel und einheitlich auf lokale Disparitäten und Bedarfe zu reagieren. Die zentrale Vorgabe von Planungsbereichen ist daher abzulösen. Der zu beplanende äußere Raum wird die KV-Region. Es werden Versorgungsstufen bestimmt und jeder Versorgungsstufe geeignete Planungsvariablen zugeordnet, mit denen jeweils Planungsräume innerhalb des insgesamt zu beplanenden Raumes der KV-Region flexibel gebildet werden können. Für eine Übergangslösung kann das Konzept der Versorgungsstufen zur Bestimmung des lokalen Versorgungsbedarfs in die Bedarfsplanungsrichtlinie aufgenommen werden.

\section{Adjustierung der Verhältniszahlen nach Demografie und Morbidität}

Kurzfristig bietet sich für die Adjustierung der historisch gefundenen Verhältniszahlen je nach Versorgungsstufe ein demografischer Korrekturfaktor an. Bei zunehmender Datentransparenz des Leistungsgeschehens und methodischer Expertise aus der Anwendung morbiditätsadjustierter Vergütungssysteme wird es gelingen, die Bandbreite einer „bedarfsgerechten Versorgung" mit bestimmenden Para- metern methodisch präziser festzulegen als dies bislang in den Konzepten gelungen ist.

\section{Planung aller Arztgruppen nach der Muster- Weiterbildungsordnung}

Derzeit werden 14 Arztgruppen in der Bedarfsplanung berücksichtigt. Nach der Musterweiterbildungsordnung könnten weit mehr geeignete Arztgruppen in die Planung aufgenommen werden, sofern die entsprechenden Voraussetzungen im SGB V geändert werden. Dabei handelt es sich überwiegend um höher spezialisierte und hoch spezialisierte Ärzte, deren Zahl häufig unterhalb der für die Planung relevanten Schwellenwerte liegen. Eine Versorgungsplanung für spezialisierte ambulante Leistungen erfordert, dass von den Landesauschüssen handhabbare Kriterien für die Bedarfsermittlung festgelegt werden. Diese sind durch Leistungsmengen und Prävalenzen bestimmbar und können weiter in Verhältniszahlen ausgedrückt werden. Die Feststellung von Leistungen und entsprechenden Verhältniszahlen, die sektorübergreifend zu planen sind, ist eine künftige Aufgabe des Gemeinsamen Bundesausschusses.

\section{Versorgungsstufen mit jeweils geeigneten Planungsinstrumenten}

Nach Patientenzugang und Leistungsspektrum gliedert sich die ambulante Versorgung in drei Versorgungsbereiche: die ärztliche Primärversorgung, die allgemeine fachärztliche Versorgung und die spezialisierte fachärztliche Versorgung bzw. die spezialisierten Leistungen. Für diese ist zur Angebotssteuerung eine weitere Differenzierung nach Vorsorgungsstufen zielführend.

\section{- Primärversorgung}

Im internationalen Kontext ist die Differenzierung der Versorgung nach Primär- oder Grundversorgung üblich, die dem Patienten einen direkten Zugang zur ersten Versorgungsstufe ermöglicht. Die Entscheidung für die weitere Versorgung wird häufig in dieser Versorgungsstufe getroffen beziehungsweise der Zugang zu weiteren Versorgungsstufen wird über den Primärversorger für den Patienten eröffnet.

Primärversorgung I: Zu dieser Versorgungsstufe werden hausärztlich tätige Ärzte (Allgemeinmediziner, Internisten, praktische Ärzte und Kinderärzte, vgl. § 73 Abs. 1a SGB V) gerechnet. Der Bedarf zur Inanspruchnahme durch den Patienten ist nach diesem Modell für diese Versorgungsstufe hoch und bedarf niedrigschwelliger Zugangskriterien.

Primärversorgung II: Ebenso geeignet für einen niedrigschwelligen Zugang, aber mit nur geringer Relevanz für die Zuweisung zu weiteren Versorgungsstufen, sind ausschließlich konservativ tätige Augenärzte und Frauenärzte. 
Abb. 2: Eckpunkte für eine differenzierte Versorgungsstufenplanung

\begin{tabular}{|c|c|c|c|c|c|c|}
\hline \multicolumn{2}{|c|}{ Regelmäßige } & \multicolumn{2}{|c|}{ Mittlere } & \multicolumn{3}{|c|}{ Krankheitsbezogene Inanspruchnahme } \\
\hline \multicolumn{2}{|c|}{$\begin{array}{c}\text { Ärztliche } \\
\text { Primärversorgung I } \\
\text { (kollektiv) }\end{array}$} & \multicolumn{2}{|c|}{$\begin{array}{c}\text { Ärztliche } \\
\text { Primärversorgung II } \\
\text { (kollektiv) }\end{array}$} & $\begin{array}{l}\text { Allg. FÄ } \\
\text { Versor- } \\
\text { gung I } \\
\text { (kollektiv) }\end{array}$ & $\begin{array}{l}\text { Allg. FÄ } \\
\text { Versor- } \\
\text { gung II } \\
\text { (kollektiv) }\end{array}$ & $\begin{array}{l}\text { Spezia- } \\
\text { lisierte } \\
\text { Leistg. } \\
\text { (optional }\end{array}$ \\
\hline Hausärzte & Kinderärzte & $\begin{array}{l}\text { Augenärzte } \\
\text { (konservativ) }\end{array}$ & Frauenärzte & $\begin{array}{l}\text { Hautärzte } \\
\text { HNO-Ärzte } \\
\text { Urologen } \\
\text { Orthopäden } \\
\text { Psychiater } \\
\text { Psycho- } \\
\text { therapie }\end{array}$ & $\begin{array}{l}\text { Anästhesis. } \\
\text { Chirurgen } \\
\text { Internisten } \\
\text { Neurologen } \\
\text { Nervenärzte } \\
\text { Radiologen }\end{array}$ & \\
\hline & & & & $\underset{\text { perspekt }}{\frac{\text { Leist }}{\mathrm{SP}}}$ & $\begin{array}{l}\frac{\text { gen gem. } \$ 115}{\text { sch auch weite }} \\
\text { ialärztliche Lei }\end{array}$ & $\begin{array}{l}116 b ; \\
\text { efinierbare } \\
\text { gen }\end{array}$ \\
\hline \multicolumn{6}{|c|}{ G-BA Richtlinie definiert bestimmende Variablen fest - Ausgestaltung/Anpassung durch Landesausschüsse } & $\begin{array}{l}\text { Länder haben } \\
\text { die Aufsicht }\end{array}$ \\
\hline \multicolumn{2}{|c|}{$\begin{array}{l}\text { - Kurze Wegezeiten } \\
10 \mathrm{~km} / 30 \text { min } \\
\text { - Kleine Planungsräume } \\
\text { - Einwohner/Arzt-Relationen } \\
\text { - Demografiegewichtung } \\
\text { - Nahverkehr / Pendlerströme } \\
\text { - Praxissitz als } \\
\text { Vollzeitäquivalente }\end{array}$} & \multicolumn{2}{|c|}{$\begin{array}{l}\text { - Mittlere Wegezeiten } \\
25-30 \text { km } \\
\text { - Mittlere Planungsräume } \\
\text { - Einwohner/Arzt-Relationen } \\
\text { - Demografiegewichtung } \\
\text { - Nahverkehr / Pendlerströme } \\
\text { - Praxissitz als } \\
\text { Vollzeitäquivalente } \\
\text { - Nur konservative Tätigkeit }\end{array}$} & $\begin{array}{l}\text { - Größere } \\
\text { Planungs- } \\
\text { räume } \\
\text { - Einwohner/ } \\
\text { Arztrelation } \\
\text { - Keine } \\
\text { Orientier- } \\
\text { ung an } \\
\text { Wegezeiten }\end{array}$ & \multicolumn{2}{|c|}{$\begin{array}{c}\text { Morbiditätsbezug } \\
\text { und QS } \\
\text { mit einheitlich } \\
\text { definierter und } \\
\text { transparenter } \\
\text { Methodik }\end{array}$} \\
\hline
\end{tabular}

Quelle: AOK- Arbeitsgruppe Bedarfsplanung

\section{- Allgemeine Fachärztliche Versorgung}

Eine allgemeine fachärztliche Grundversorgung sollte sich im Idealfall an die Grundversorgung anschließen. Der Patientenzugang erfolgt aber häufig direkt. Die Inanspruchnahmehäufigkeit ist gegenüber den Primärversorgern geringer, so dass von der Primärversorgung abweichende Planungsparameter notwendig sind.

Allgemeine fachärztliche Versorgung I: Die Planungsparameter für die Fachärztliche Versorgung I orientieren sich an den Häufigkeiten der Inanspruchnahme und dem Versorgungsbedarf. Zu dieser Versorgungsstufe werden alle bereits in der Bedarfsplanung relevanten Facharztgruppen gerechnet: Hautärzte, HNO-Ärzte, Urologen, Orthopäden, Psychiater und Psychotherapeuten.

Allgemeine Fachärztliche Versorgung II: Die zweite fachärztliche Versorgungsstufe umfasst Internisten mit ihren jeweiligen Schwerpunktbezeichnungen, die als eigenständige Arztgruppen zu planen sind. Zusätzlich werden Anästhesisten und allgemein operierende Chirurgen dieser Versorgungsstufe zugerechnet, wie auch Neurologen und Radiologen. Der Zugang zu diesen Arztgruppen bzw. Ärzten wie auch die Inanspruchnahme ist an gut abgrenzbaren Indikationen orientiert oder wird überwiegend von den vorangegangenen Versorgungsstufen veranlasst.

\section{- Spezialisierte Fachärztliche Versorgung bzw. spezialisierte Leistungen}

Die dritte fachärztliche Versorgungsstufe wird im Folgenden als „dritter Sektor“ betrachtet, da er sowohl fachärztliche ambulante Leistungen umfasst, als auch die ambulante Behandlung im Krankenhaus (vgl. 3. Abschnitt; viertes Kapitel SGB V). Die Leistungen zeichnen sich, bezogen auf die jeweils betrachtete Population, durch eine geringe bis seltene Inanspruchnahme aus, erfordern eine hohe Expertise bzw. eine Teamorientierung der Behandlung und einen Aufwand, der zum Teil nur im stationären Bereich vorgehalten werden kann. Allen Leistungen gemein ist die fehlende Bedarfs- oder Versorgungsplanung bzw. fehlende 
Parameter, die diese ermöglichen könnten. Der „dritte Sektor" beinhaltet Leistungen, die nach Möglichkeit jeweils sektorunabhängig mit leistungsbezogenen Versorgungsaufträgen auszustatten sind.

\section{Planungsparameter für die Versorgungsstufen}

\section{- Primärversorgung}

Die Vermeidung einer Fehlallokation von Primärversorgern ist in der gegenwärtigen Bedarfsplanungssystematik nicht möglich, da die Planungsbezirke mehrere Gemeinden bzw. Postleitzahlenregionen umfassen. Parameter, die geeignet sind, eine niedrigschwellige Erreichbarkeit der Primärversorgungsstufe zu ermöglichen, werden durch Wegezeiten, Entfernungen und Anbindung an den öffentlichen Nahverkehr abgegrenzt. Die Planungsräume werden dabei auf Landesebene durch die Landesausschüsse beschrieben. Die bestehenden Verhältniszahlen bleiben erhalten und werden mit demografischen Informationen korrigiert. Die Anwendung wird auf Planungsräume kleinerer Größe modifiziert. Die Zulassung erfolgt, sofern möglich, ortsgebunden.

\section{- Fachärztliche Versorgungsstufe I und II}

Die fachärztliche Versorgung wird in allen Versorgungsstufen Bestandteil der sektorübergreifenden Rahmenplanung. Je nach Versorgungsstufe sind die Planungsräume kleiner oder größer zuzuschneiden. Die ortsgebundene Ansiedlung kann nicht sichergestellt werden, wäre aber wünschenswert. Eine angemessene Erreichbarkeit dieser Versorgungsstufe orientiert sich für die fachärztliche Versorgung I an Planungsräumen mittlerer Größe, für die fachärztliche Versorgung II und die spezialisierten Leistungen am KV-Bezirk.

Die Planungsräume werden durch die Landesausschüsse beschrieben. Verhältniszahlen werden neu bestimmt und auf große flexible Planungsräume bezogen. Die Planung erfolgt grundsätzlich mit einer sektorübergreifenden Analyse und entsprechenden Zulassungen. Für die Arztgruppen der Fachärztlichen Versorgung II ist aufgrund des ausgeprägten Indikationsbezugs der Inanspruchnahme bzw. des Zugangs zur Versorgung eine morbiditätsbezogene Bedarfsermittlung angemessen.

\section{Zulassung}

Das Zulassungssystem wird rechtlich sektorunabhängig vereinheitlicht und kann den Versorgungsbedarfen nach inhaltlich adäquat differenziert bzw. gebunden werden:

- „Zulassungen“ und „Ermächtigungen“ zur ambulanten Versorgung werden als Zulassungen zusammengefasst. Leistungserbringer (ambulant und stationär), die im Bereich der fachärztlichen Versorgung tätig sein möchten, können beim künftig sektorübergreifend aufgestellten Zulassungsausschuss eine Zulassung beantragen. Diese gilt für Ärzte, die die allgemeinen Zulassungsvoraussetzungen gemäß § 95 Abs. 2 SGB V erfüllen, für zugelassene Vertragsärzte und Krankenhausärzte gleichermaßen.

- Ein Versorgungsauftrag kann auf konkrete Leistungsbereiche, Leistungsinhalte oder ein bestimmtes Leistungsvolumen begrenzt und zeitlich befristet werden. Dieser richtet sich für die fachärztlichen Leistungen II sowie die spezialisierten Leistungen nach dem morbiditätsorientierten und qualitätsbezogenen Bedarf. Beantragt

Tabelle 1: Planungsparameter für die Versorgungsstufen

\begin{tabular}{|l|l|l|l|l|l|}
\hline Planungsparameter & $\begin{array}{l}\text { Primär- } \\
\text { versorgung I }\end{array}$ & $\begin{array}{l}\text { Primär- } \\
\text { versorgung II }\end{array}$ & $\begin{array}{l}\text { Fachärztliche } \\
\text { Versorgung I }\end{array}$ & $\begin{array}{l}\text { Fachärztliche } \\
\text { Versorgung II }\end{array}$ & $\begin{array}{l}\text { Spezialisierte } \\
\text { Leistungen }\end{array}$ \\
\hline $\begin{array}{l}\text { Flexible Planungsräume: } \\
\begin{array}{l}\text { Feststellung durch } \\
\text { Landesausschüsse }\end{array}\end{array}$ & Kleine Räume & Mittlere Räume & Große Räume & Große Räume & Große Räume \\
\hline Kurze Wegezeiten/Erreichbarkeit & $\begin{array}{l}10 \mathrm{~km} \\
30 \mathrm{~min}\end{array}$ & $\begin{array}{l}25-30 \mathrm{~km} \\
30 \mathrm{~min}\end{array}$ & Nein & Nein & Nein \\
\hline Neue Einwohner/Arzt-Relationen & Ja & Ja & Ja & Nein & Nein \\
\hline Demografiegewichtung & Ja & Ja & Nein & Nein & Nein \\
\hline Morbiditätsgewichtung & Ja & Ja & Ja & Ja \\
\hline $\begin{array}{l}\text { Berücksichtigung von } \\
\text { Öffentlichem } \\
\text { Nahverkehr/Pendlerströmen }\end{array}$ & Ja & Ja & Nein & Ja & Nein \\
\hline Ortsbindung zur Zulassung & & Nein & Ja & Jein \\
\hline Sektorübergreifend & & Jain & \\
\hline
\end{tabular}

Quelle: AOK- Arbeitsgruppe Bedarfsplanung 
werden können Leistungen für einen vollen oder hälftigen Versorgungsauftrag.

Nach diesem Konzept wird künftig auch für den Versorgungsbereich der spezialisierten Leistungen eine bisher fehlende Bedarfs- bzw. Versorgungsplanung geschaffen. Versorgungsaufträge können leistungsbezogen formuliert und zeitlich gebunden werden. Mit einer leistungsgebundenen Feststellung neuer Verhältniszahlen wird es den Aufsichtsbehörden möglich, von den Krankenkassen mit unterschiedlichen Vertragsformen kontrahierte Leistungsmengen für die Versorgungsplanung zu bilanzieren. Dabei können wettbewerbliche Verträge der Krankenkassen flexible Sicherstellungspartnerschaften friktionsfrei einbezogen werden.

\section{Ambulante Beteiligung der Krankenhäuser mit Qualitätsvorrang}

\section{Doppelstrukturen der ambulanten Versorgung}

Für die Beteiligung der Krankenhäuser an der ambulanten Versorgung wurden von der Politik in langjährigen Prozessen versorgungspolitisch immer wieder gute Gründe angeführt. Mit den jeweils geschaffenen Formen verbindet sich indessen bis heute kein stimmiges Konzept. Experten sprechen von einem Flickenteppich, den es konzeptionell zu ordnen gilt. Heute bestehen nebeneinander:

vor- und nachstationäre Behandlung im Krankenhaus (§ 115a SGB V ),

- teilstationäre Behandlung im Krankenhaus (§ 39 SGB $\mathrm{V})$,

- die Hochschulambulanzen (§ 117 SGB V),

- das ambulante Operieren ( $(115 b$ SGB V),

- die Behandlung von seltenen Erkrankungen und Krankheiten mit besonderem Krankheitsverlauf ( $§ 116 \mathrm{~b}$ SGB $\mathrm{V})$,

- die psychiatrischen Institutsambulanzen (§ 118 SGB V),

- die Sozialpädiatrischen Zentren (§ 119 SGB V),

- die pädiatrischen Spezialambulanzen (§ 120 Abs. 1a SGB V),

- ambulante Ermächtigungen von Krankenhausärzten (§ 116 ff. SGB V),

- ambulante Versorgung bei Unterversorgung (§ 116a SGB $\mathrm{V})$,

- die Behandlung im Rahmen der DMP (§ 116b Abs. 1 SGB V)

- und nicht zuletzt die Integrierte Versorgung (§ 140a ff. SGB V).

Wie in Abschnitt 2 gezeigt wurde, ermöglicht eine neue Versorgungsplanung vom Grundsatz her den systematischen Einbezug der Krankenhäuser. Der Schlüssel dafür ist bei der spezialärztlichen Versorgung ein künftiger Leistungsbezug der Planung. Damit entsteht ein Gesamtbild für die sektorunabhängige Sicherstellung der Versorgung. Entsprechend müssen auch passende Vertrags- und Vergütungskonzepte sowie Vereinbarungen zur Mengensteuerung aufgesetzt werden. Es ist auf Dauer nicht vertretbar, dass die vertragsärztliche Versorgung, die notwendigerweise Regulierungen unterliegt, von einem unübersichtlichen und kaum steuerbaren Flickenteppich ambulanter Behandlung im Krankenhaus ergänzt wird. Die versorgungspolitische Bedeutung dieses Teils der ambulanten Versorgung ist unbestritten. Ebenso unbestritten ist, dass der Einbezug der Krankenhäuser und Institute in die genossenschaftliche Verwaltung der Vertragsärzte systemfremd wäre. Von daher müssen

- die sektorübergreifende Planung Bedarfsanalysen einbeziehen,

- die Zulassungen leistungsbezogen und ggf. zeitlich gebunden erfolgen

- und eine qualitätsorientierte vertragliche Mengensteuerung eingefordert werden.

\section{Spezialärztliche Leistungen sektorübergreifend kontra- hieren}

Versorgungsinhaltlich sind die Krankenhäuser ambulant bei spezifischen Leistungen beteiligt, die zur spezialärztlichen Versorgung gehören. Dazu zählen insbesondere Leistungen nach § 115b SGB V (ambulantes Operieren), § 116b SGB V (seltenen Erkrankungen, besonderer Krankheitsverlauf) und § 118 SGB V (psychiatrische Hochschulambulanzen). Nach dem Konzept des AOK-BV zur künftigen Bedarfsplanung soll der Gemeinsame Bundesausschuss Schritt für Schritt die Voraussetzungen dafür schaffen, dass die spezialärztlichen Leistungen in die sektorübergreifende Planung, Qualitätssicherung und Vertragsgestaltung überführt werden können. Dazu sind die Leistungen basieren auf Leitlinien nach den Maßstäben der evidenzbasierten Medizin

- hinsichtlich ihrer Indikationsanforderungen und jeweiligen Nutzenerwartung bzw. des Zusatznutzens gegenüber den relevanten therapeutischen Alternativen zu beschreiben,

- hinsichtlich ihrer Qualitätskriterien und geeigneter Qualitätssicherungsmaßnahmen einzuordnen und

- hinsichtlich ihres populationsbezogen erwarteten Bedarfs in der sektorübergreifenden Versorgung auch anhand internationaler Referenzdaten zu bewerten.

Auf dieser Grundlage sollen neu aufgestellte, paritätische Landesausschüsse nach § 90 SGB V mit auf der Leistungsanbieterseite gemeinschaftlichem Stimmrecht von KV/ LKG bei sektorübergreifenden Angelegenheiten, für die jeweiligen Leistungen regionale Bedarfe versorgungsinhaltlich populationsbezogen feststellen und Verhältniszahlen ggf. entsprechend regional adjustierten. 


\section{Vertrags- und Qualitätssicherung für Spezialärztliche Leistungen}

\section{Bedarfsgerechte Versorgung}

Wenn sich Vertragsärzte und Krankenhäuser sektorübergreifend um leistungsbezogen geplante Versorgungsaufträge bewerben, stellt sich insbesondere in strukturell überversorgten Bezirken die Frage der Bedarfszulassung. Gründe aber, an dieser Stelle nicht nur bedarfsorientierte, sondern auch versorgungsinhaltliche und qualitative/ zeitliche Bindungen vorzusehen, ergeben sich allein schon aus der fortschreitenden Spezialisierung und dynamischen Wissensentwicklung in der Medizin. Leistungsbezogene Versorgungsaufträge verlangen geradezu nach einer unmittelbaren, mit Rückkopplungsprozessen verbundenen Qualitätssicherung, wie sie etwa bereits beim Mammographie-Screening vorbildlich eingeführt worden ist. Wer außerhalb der Toleranzen unzutreffende Befunde erhebt, wird sich hier nicht auf seine Zulassung berufen können. Von daher wird deutlich, dass leistungsbezogene Versorgungsaufträge von der rechtlichen Form der Zulassung getrennt betrachtet werden müssen. Die Frage der Bedarfszulassung kann daher dahin stehen, wenn etwa leistungsbezogene Verträge mit zeitlichen Bindungen verknüpft werden.

Dem Gesetzgeber steht es frei - wie bei der Hilfsmittelversorgung ( 127 SGB V) mit Erfolg praktiziert - vom Zulassungs- auf das Vertragsprinzip überzugehen und vorzuschreiben, dass die Leistung von ihren Ergebnissen her zu beurteilen ist und nicht primär danach, ob der Erbringer zugelassen ist. Jedenfalls hat der tradierte Ansatz, alle Leistungserbringer zuzulassen, um mit Formen der nachgehenden Preissteuerung auf ein medizinisch begründbares und finanzierbares Ergebnis hinzuwirken, zu extensiver Versorgung mit hohem Ressourcenverbrauch geführt, ohne dass damit eine dem entsprechend hohe Qualität für die Versicherten und Berufszufriedenheit der Ärzte einherginge. Denn entgegen der ökonomischen Logik führt ein Nachgeben der Preise aufgrund steigender Leistungsfrequenzen zu einem neuen Anreiz, die Zahl der abgerechneten Leistungen zu erhöhen. Für die spezialärztlichen Leistungen sollte dagegen die medizinische Angemessenheit und Qualität der Leistung als vorrangiges Kriterium festgelegt werden können. Diese Form der Mengenbegrenzung ist medizinisch rational.

\section{Bedarfsgerechte Vergabe von Versorgungsaufträgen}

Eine Grundlage dafür könnte durch die sektorübergreifende Ausschreibung der Leistungen unter Vorrang von Qualitätskriterien gelegt werden. Dabei greifen die Krankenkassen für den jeweiligen Planungsbezirk einheitlich und gemeinsam auf der Grundlage der Bedarfsfeststellungen des Landesausschusses insbesondere auf die Qualitätskriterien des Bundesausschusses zurück, um Angebote zu bewerten. Es werden unter einheitlicher Gewichtung der Kriterien so viele Angebote berücksichtigt, dass der erwartete Bedarf erfüllt wird. Würde der Gesetzgeber diesen zu einem Qualitätsvorrang wählen, könnten die Krankenkassen gemeinsam Versorgungsaufträge mit hinreichender Dauer vergeben. Der Leistungsbezug der spezialärztlichen Versorgungsaufträge stellte dabei zum einen in der Regel sicher, dass die Nichtberücksichtigung eines Anbieters weite Teile seiner Gesamttätigkeit unberührt ließe. Zum anderen ist ein Niederlassungszwang von Hochspezialisten gesundheitspolitisch nicht opportun. An dem erforderlichen rechtlichen Spielraum mangelte es dem Gesetzgeber an dieser Stelle nicht.

Ginge der Gesetzgeber diesen Weg nicht, sollte er zumindest die nachstehenden wettbewerblichen Vertragsoptionen des SGB V auf die neue sektorübergreifende Bedarfsplanung und die sich daraus ergebende Vertragslandschaft hin anpassen bzw. erweitern. Die vergaberechtlichen Grundlagen dazu sind mit dem AMNOG bereits auf das Kartellrecht ausgedehnt worden.

\section{Vertragslösungen unter Qualitätsvorrang}

\section{Erweiterung selektiver Verträge (neuer § 73d SGB V)}

Nach den Besonderheiten der jeweiligen Leistungen, der unterschiedlichen Beteiligung von Angebotsformen und den regionalen Gegebenheiten, unter denen eine vom Bedarf her definierte Vergabe von Versorgungsaufträgen erfolgt, sind jeweils adäquate vertragliche Initiativen zur besonderen Qualitätssicherung der spezialärztlichen Leistungen zu gestalten. Dazu sollte zumindest die Möglichkeit geschaffen werden, für die leistungsbezogen und sektorübergreifend geplanten ambulanten spezialärztlichen Leistungen auch mit Krankenhäusern Selektivverträge abschließen zu können. Aus Sicht der AOK sollte hierfür ein neuer „§ 73d SGB V“ nach dem Vorbild des § 73c SGB V geschaffen werden, in dem sowohl die Vertragsärzte, als auch die Krankenhäuser als mögliche Vertragspartner der Selektivverträge für diese ambulanten spezialärztlichen Leistungen vorgesehen werden. Allerdings sollte aus Gründen der Entbürokratisierung immer dann, wenn Verträge zu spezialärztlichen „Leistungen“ betroffen sind, gesetzlich auf die Anforderung nach Einschreibung des Versicherten verzichtet werden.

\section{Direktvertrag ohne parallelen Kollektivvertrag}

Selektive Verträge ermöglichen es, von den Regelungen des 4. Kapitels des SGB V abzuweichen. Angesichts zentraler Finanzzuweisungen an die Krankenkassen und des verbleibenden Kontrahierungszwangs gegenüber allen Anbietern erfordern die mit Selektivverträgen verbundenen Doppelstrukturen allerdings aufwendige Regulierungen, um Doppelzahlungen und oft nicht kalkulierbare finanzielle Risiken zu vermeiden. Dies limitiert den möglichen Erfolg auf Seiten der Vertragspartner und den Nutzen der Versicherten. Soll von daher nicht schon grundständig in Kauf genommen werden, dass Einzelverträge Doppelstrukturen schaffen, ist gesetzlich eine Ausstiegsoption der Krankenkasse aus dem Kollektivvertrag zu ermöglichen. 


\section{THEMA}

Über den bisherigen Interimsansatz zu „selektiven“ Verträgen hinaus sollte in diesem neuen § 73d SGB V für die jeweilige Krankenkasse gesetzlich eine zusätzliche Option eröffnet werden, bei sektorübergreifenden spezialärztlichen Leistungen regional ein Nebeneinander von Kollektiv- und Selektivverträgen grundsätzlich zu vermeiden. Dazu ist gesetzlich eine ergänzende einzelvertragliche Option für die Krankenkasse einzuräumen, nach der sie die jeweiligen spezialärztlichen Leistungen in einem Planungsbezirk abschließend ausschreiben kann. Ein Kontrahierungszwang entfällt. Die kollektivvertraglichen Vereinbarungen werden für den Planungsbezirk ausgesetzt. Dadurch können in diesem Bereich „situative“ Einschreibungen und fortgesetzte Bereinigungen der Gesamtvergütung vermieden werden. Die kollektive Gebührenordnung bildete in diesem Falle Höchstpreise ab, die aufgrund besonderer vertraglicher Qualitätsvereinbarungen überschritten werden können; die Qualitätsanforderungen des Gemeinsamen Bundesausschusses stellen Mindeststandards dar. Kurzum: Die ordnungspolitischen Maßstäbe aus Leistungskatalog, Bedarfsplanung, Qualitätssicherung, Gebührenordnung und Wirtschaftlichkeitsprüfung bleiben bestehen, soweit nicht weitergehende Regelungen vereinbart sind. Damit würde für den Vertragswettbewerb unter Qualitätsvorrang eine nachhaltige Entwicklung ermöglicht. Entwicklungen der AOK zu Qualitätsindikatoren für die ambulante und stationäre Versorgung lassen sich auch auf spezialärztlichen Leistungen übertragen und können in die Vertragsgestaltung einbezogen werden.

Bei einer Ausschreibung einzelnen Krankenkassen bzw. Einkaufsgemeinschaften wären die Krankenkassen im Vorteil, die inhaltlich und in dem jeweiligen Planungsraum eine adäquate Ausschreibung realisieren können. In anbetracht der Marktstrukturen ist die Zahl handlungsfähiger Akteure in dem Feld kaum anders zu beurteilen als bei der Ausschreibung von Arzneimitteln. Und angesichts des Ziels eines flächendeckend zugänglichen Angebots wäre auch nicht zu erwarten, dass einzelne ausschreibende Krankenkassen ihre Nachfrage unverhältnismäßig konzentrierten. Nur in diesem Falle hätten Konkurrenten überhaupt Vertragsengpässe zu befürchten.

Ebenso verbleiben Krankenkassen, die aufgrund ihrer Struktur oder von Präferenzen der Versicherten den Kollektivvertragsweg vorziehen oder unternehmerische Wagnisse als prohibitiv einschätzen, trotz der regionalen Wettbewerbsoption im Kollektivvertrag. Diejenigen Versicherten/ Patienten, Ärzte/Zentren und Krankenkassen, die sich zu Direktverträgen finden und die Vorteile nutzen wollen, können ohne jede Beeinträchtigung für den Kollektivvertrag für die spezialärztliche Versorgung das Neue wagen, wenn sie sich unternehmerisch darauf vorbereiten.

\section{Marburger Schriften zum Gesundheitswesen}

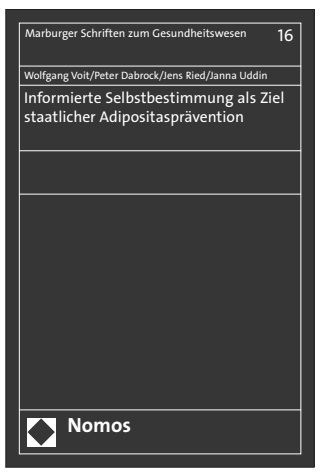

Informierte Selbstbestimmung als Ziel staatlicher Adipositasprävention

Herausgegeben von Prof. Dr. Wolfgang Voit, Prof. Dr. Peter Dabrock, M.A., Dr. Jens Ried und Janna Uddin 2011, Band 16, ca. 150 S., brosch., ca. 37,- $€$ ISBN 978-3-8329-6283-8 Erscheint ca. April 2011

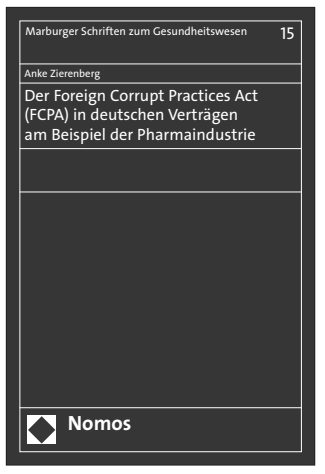

Der Foreign Corrupt Practices Act (FCPA) in deutschen Verträgen am Beispiel der Pharmaindustrie Von RAin Dr. Anke Zierenberg 2011, Band 14, ca. 420 S., brosch., $99,-€$ ISBN 978-3-8329-6341-5 Erscheint ca. April 2011

Bitte bestellen Sie im Buchhandel oder versandkostenfrei unter $\mathbf{w w w}$.nomos-shop.de

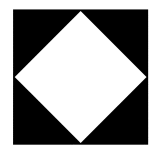

Nomos 